\title{
Does it matter to define what is matter? \\ A primer for materialists and beyond
}

\author{
Patrizio Tressoldi $* 1$ \\ *Science of Consciousness Research Group, Studium Patavinum, Padova University, \\ Italy
}

[version $6 / 2 / 2021]$

\begin{abstract}
The definition of what is the matter is fundamental not only for those supporting a materialist point of view of reality including our mental experiences, but also for those who support an alternative view.

Starting from a brief historical overview of the ideas of what is the matter up to the more recent scientific theoretical and empirical contributions to this definition, we will show that the foundations of Materialism and Realism are falsified and must be replaced by an alternative interpretation of reality and, consequently, of who we really are.

Western Idealism and Eastern Adavaita-Vedanta fits well with the modern evidence of what is the matter ad suggest Consciousness as the basic stuff of all that exists.
\end{abstract}

Keywords: materialism; realism; idealism; quantum physics; perception; consciousness

${ }^{1}$ corresponding author: patrizio.tressoldi@unipd.it 


\section{Introduction}

The aim of this paper is to discuss what is matter which is considered by Materialism as the basic stuff of all that exists: objects and events, including our consciousness states and their contents.

Through a brief overview of the main Western and Eastern philosophical interpretations of matter to the updated experimental findings from particle and quantum physics, we will try to give the readers a clear explanation of what is the matter according to the more recent scientific interpretations.

Trying to define what is the matter will inevitably bring us to discuss what is reality and the philosophy of Realism. Realism, in its pure form, postulates that there is a reality, that is, things, that are independent from who perceives them (Miller, 2019).

In summary, with this paper we aim to stimulate a constructive debate on these two fundamental questions:

-What is the matter?

-Is it independent from who perceives it?

\section{What is the matter: a brief historical overview}

What is the matter was one of the more important topics for the Greek philosophers. According to Aristotle, matter was the outcome of different combinations and interactions of four basic elements: earth, air, water and fire. Differently, other Greek philosophers, for example, Leucippus, Democritus, Epicurus and Lucretius, supported the view that matter was made of atoms, that is "uncuttable" things having a form and occupying a space (Berryman, 2016).

Differently, in some Indian philosophical traditions, for example in the Sāmkhya philosophy, prakriti is the primaeval stuff out of which all things arose and has the following properties: uncaused, eternal, all-pervading, independent, self-complete and with no distinguishable parts.

It is interesting that prakriti has no spatial boundaries with respect to the idea of the atom which represents the smallest part of matter.

We will see that the scientific investigation has been pursuing the same path, that is, discover the smallest, "uncuttable" part of what can be defined as matter. 


\section{What is the matter: an update from particle and quantum physics}

The history of the scientific search for the ultimate components of matter, that is, of the atom is too long to be described here. For those interested in this history, we suggest these two websites: https://www.timetoast.com/timelines/history-of-the-atomic-model--36 and https://medium.com/@Intlink.edu/a-timeline-of-atomic-models-cb2607b1da85

What emerges from this history is that the search for an "uncuttable" particle with well-defined mass and location that occupies a precise space has vanished completely, leaving us with strange objects that are still named particles, but are without several characteristics we use to define an object in our real life. In Table 1, we compare the main differences between classical and quantum "objects"

Table 1: Main differences between classical and quantum "objects"

\begin{tabular}{l|c|c|}
\hline \multicolumn{1}{c|}{ Characteristics } & Classical & Quantum \\
\hline position & exact & probabilistic \\
\hline speed & exact & probabilistic \\
\hline either a particle or a wave & yes & no \\
\hline $\begin{array}{l}\text { independent from the } \\
\text { observer }\end{array}$ & yes & acausal \\
\hline $\begin{array}{l}\text { interaction } \\
\text { change }\end{array}$ & causes precede the effects & quantised \\
\hline
\end{tabular}

However, even if there is a general consensus on what a particle is not, there are multiple different opinions on what a particle is. In an interview, Wolchover (2020), described seven different interpretations supported by different physicists: from a collapsed wave form to a deformation of an underlying qubit field and to what we measure in detectors. But this list is very probably not complete (see Hobson, 2013).

\section{Is the Moon there when nobody looks?}

To make things more intriguing, what emerges from the scientific theoretical and empirical contributions is that the act of observing/measuring what is a particle, that is whatever it is, contributes to its reality. In other words, it seems that one of the critical characteristics of an object, its existence (reality) independent from who observes it, as postulated by Realism (Miller, 2019) is not more valid or heavily questioned.

"Is the moon there when nobody looks? Reality and the quantum theory" is the famous title of a paper written by David Mermin in 1985 (Mermin, 1985) which was inspired by a personal account of Abrahan Pais of a discussion he had with Albert Einstein: "We often discussed his notions on objective reality. I recall that during one walk Einstein suddenly 
stopped, turned to me and asked whether I really believed that the moon exists only when I look at it” (Pais, 1979, pag. 907).

This problem is still hotly debated both at the theoretical and empirical level and not only within the physics community. For example, in 2015, Howard Wiseman discussing the experimental findings of Hensen et al. (2015) wrote a paper entitled "Death by experiment for local realism" and the core argument was: "Fundamental scientific assumption called local realism conflicts with certain predictions of quantum mechanics. Those predictions have now been verified, with none of the loopholes that have compromised earlier tests" (Wiseman, 2015).

The list of experimental evidence is large and continues to grow, e.g. "Violation of local realism with freedom of choice" by Scheidl et al. 2010; "Strong loophole-free test of local realism" by Shalm et al. 2015; "Challenging local realism with human choices" by The BIG Bell Test Collaboration 2018, etc.

Even if the scientific evidence which falsify realism seems quite convincing, we can object that this violation is valid only at a microscopic level. Does it also apply to an apple, a table and every other object we can perceive by our sensory organs? At this point a critical question arises: is reality what we detect/perceive with our sensory organs and processed by our brain or it is only complex filter of an underlying reality? How will an apple appear to an eagle or to a snake? How does reality change when we modify the constraints of our sensory organs using modern microscopes and telescopes?

See for example Figure 1 in order to have an idea of how large the wavelength spectrum is and, hence, what we could know by simply enlarging the range of information we can perceive.

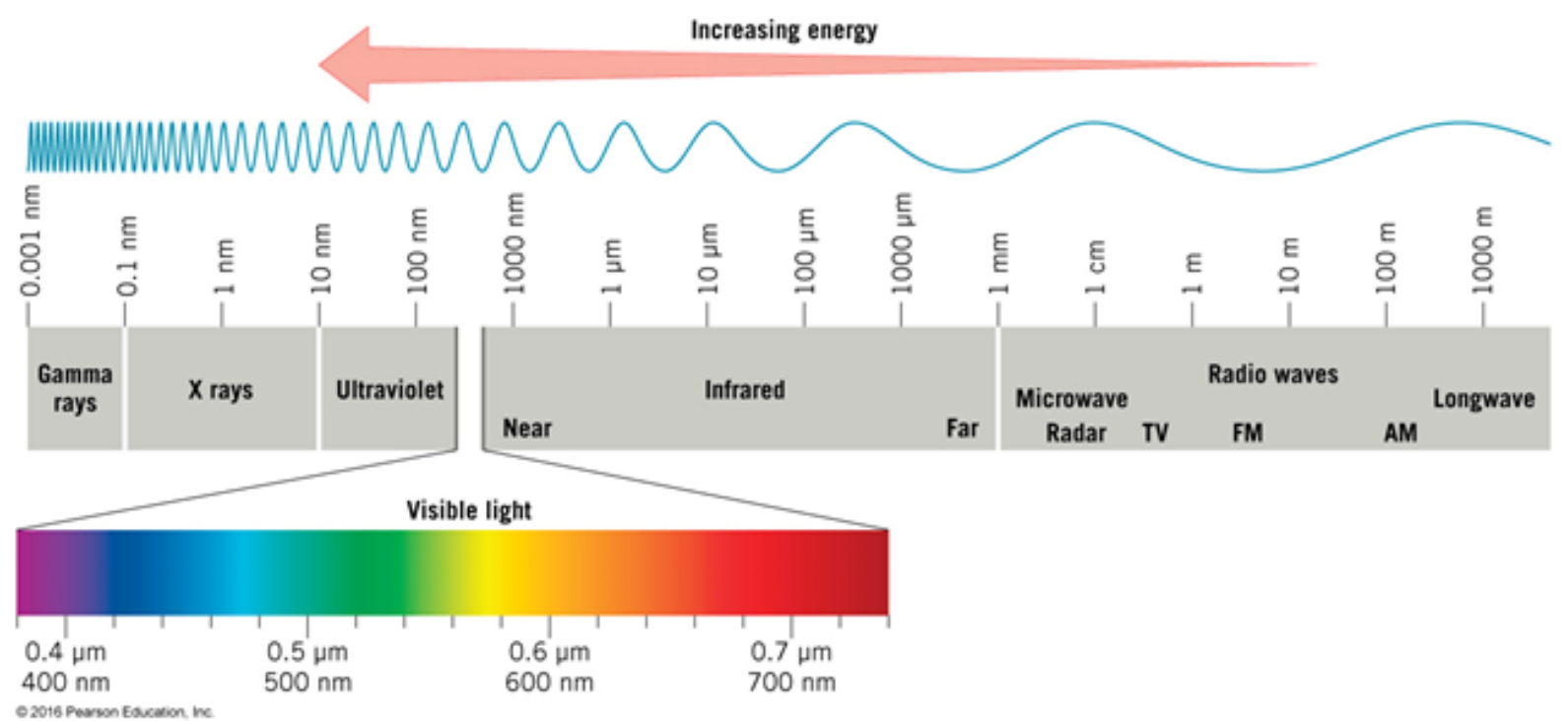

Figure 1: wavelength spectrum example. Credits http://www.faculty.luther.edu/ bernatzr/Courses/Sci123/Chapter02 
How would the world appear if we could expand the range of our visual, auditory, olfactory and tactile information to the smallest and the largest ultimate components that allow us to define what is reality out there?

If we follow this "gedanken experiment" (thought experiment), it becomes clear that matter and the reality of things are nothing but perceptions, that is "mental stuff" filtered by our sensory organs. In other words, matter and reality are not outside, but only inside our mind/consciousness.

This interpretation fits well with the Interface Perception Theory (IPT) of Donald Hoffman (Hoffman, Singh, \& Prakash, 2015; Prakash, Stephens, Hoffman, et al. 2020). The basic assumption of the IPT is that human, as well as animal perceptual systems, have evolved to provide a species-specific interface to guide adaptive behavior, and that this interface model demonstrates the Fitness-Beats-Truth (FBT) theorem, that is that a veridical representation of objective reality is less adaptive.

\section{Conclusions}

What we perceive in our ordinary awake state of consciousness as objects made of matter that occupy a well-defined space outside our body with well precise properties, like colors, forms, velocity, etc., is nothing but perceptual (mental) information generated by our perceptual systems, acting as filters with respect to an underlying ultimate reality. However, even if mental instead of material, this type of information is very useful in allowing us to live, as supported by the IPT.

If what we naively consider matter is nothing but mental information created by our sensory organs, if we modify their functioning or if we bypass them, we can obtain different types of mental information. This is exactly what happens when the sensory organs and/or the neural activity of the brain associated with the elaboration of the information received by them is damaged or altered, for example, with the application of external electric or magnetic stimulations, such as transcranial direct current stimulation and transcranial magnetic stimulation, or by the ingestion of natural or artificial substances such as psilocybin and serotonin reuptake inhibitors.

Furthermore, by bypassing the sensory organs, for instance in an out-of-body state of consciousness (Tressoldi et al., 2015; Nicholls, Pederzoli \& Tressoldi, 2019; Ballati et al., 2020) or by the use of nonlocal perception techniques (Tressoldi \& Storm, 2021; Tressoldi \& Storm 2020), it becomes possible to obtain information which violate the perceptual constraints of our sensory organs. For example, when in an out-of-body state of consciousness, participants have referred to being able to see objects or scenes from all points of view simultaneously, an ability not possible with our visual system (Tressoldi et al., 2015).

The above phenomena plus the nature of qualia, that is the phenomenological personal experiences, and mind-body causal effects, like placebo, analgesic hypnosis, etc., 
give support to the hypothesis that Consciousness is the primary stuff of all that exists (see Tressoldi \& Facco, 2021).

This interpretation fits well with Western Idealism (Kastrup, 2018; Guyer \& Horstmann, 2021), which postulates that reality itself is a form of great thought/consciousness and that human thoughts participate in it and Eastern AdavaitaVedanta philosophy which means non-duality (Indich, 1995; Wikpedia, 2021) which states that the only reality is Brahman, the "pervasive, infinite, eternal truth and bliss which does not change, yet is the cause of all changes", which we can also be defined as the "primordial or pure Consciousness".

What is particularly interesting is that this primary Consciousness can be experienced with advanced meditation practices (Josipovic, 2021; Wahbeh et al. 2018) and mystical/transcendental experiences (Facco, Lucangeli \& Tressoldi, 2019; Yaden et al. 2016). 
Acknowledgements: I thank Marco Genovese, for his supervision about the quantum physics information. I also thank the Proof-Reading-Service for English revision. 


\section{References}

Ballati, A., Prati, E., Pederzoli, L., Eng, M., \& Tressoldi, P. (2020). Remote Viewing with and without controlled Out-Of-Body Consciousness. Advanced Research in Psychology, https://doi.org/10.46412/001c.14154

Berryman, S. (2016). Ancient Atomism. The Stanford Encyclopedia of Philosophy. Edward N. Zalta (Ed.), https://plato.stanford.edu/archives/win2016/entries/atomism-ancient .

Facco, E., Lucangeli, D., \& Tressoldi, P. (2019). Dr. AM-A case of a modern mystic? Implications for psychology and medicine. Spirituality in Clinical Practice, 6(1), 44. DOI: $10.1037 / \mathrm{scp} 0000171$

Guyer, P. \& Horstmann, R-P. (2021). Idealism. The Stanford Encyclopedia of Philosophy (Spring 2021 Edition), Edward N. Zalta (ed.), forthcoming https://plato.stanford.edu/archives/spr2021/entries/idealism

Hensen, B., Bernien, H., Dréau, A. E., Reiserer, A., Kalb, N., Blok, M. S., ... \& Hanson, R. (2015). Loophole-free Bell inequality violation using electron spins separated by 1.3 kilometres. Nature, 526(7575), 682-686.

Hobson, A. (2013). There are no particles, there are only fields. American Journal of Physics, 81(3), 211-223. http://dx.doi.org/10.1119/1.4789885

Hoffman, D. D., Singh, M., \& Prakash, C. (2015). The interface theory of perception. Psychonomic Bulletin \& Review, 22(6), 1480-1506. https://doi.org/10.3758/s13423-015$\underline{0890-8}$

Indich, W. M. (1995). Consciousness in Advaita Vedanta. Motilal Banarsidass Publisher Kastrup, B. (2018). The universe in consciousness. Journal of Consciousness Studies, 25(56), 125-155.

Josipovic, Z. (2021). Implicit-explicit gradient of nondual awareness or consciousness as such. MindRxiv https://mindrxiv.org/ngupr

Mermin, D.N. (1985). Is the moon there when nobody looks? Reality and the quantum theory Physics Today, 38-47

Miller, A. (2019). "Realism", The Stanford Encyclopedia of Philosophy (Winter 2019 Edition), Edward N. Zalta (ed.), https://plato.stanford.edu/archives/win2019/entries/realism

Nicholls, G., Pederzoli, L., \& Tressoldi, P. (2019). The phenomenology of spontaneous and hypnotically induced Out-of-Body Experiences: A comparison. PsyArXiv; https://doi.org/10.31231/osf.io/pqcer

Pais, A. (1979). Einstein and the quantum theory. Review of Modern Physics, 51, 863-914. 
Prakash, C., Stephens, K. D., Hoffman, D. D., Singh, M., \& Fields, C. (2020). Fitness beats truth in the evolution of perception. Acta Biotheoretica, 1-23. https://doi.org/10.1007/s10441020-09400-0

Shalm, L. K., Meyer-Scott, E., Christensen, B. G., Bierhorst, P., Wayne, M. A., Stevens, M. J., ... \& Nam, S. W. (2015). Strong loophole-free test of local realism. Physical Review Letters, 115(25), 250402.

Scheidl, T., Ursin, R., Kofler, J., Ramelow, S., Ma, X. S., Herbst, T., ... \& Zeilinger, A. (2010). Violation of local realism with freedom of choice. Proceedings of the National Academy of Sciences, 107(46), 19708-19713.

www.pnas.org/cgi/doi/10.1073/pnas.1002780107

The BIG Bell Test Collaboration., Abellán, C., Acín, A. et al. (2018). Challenging local realism with human choices. Nature 557, 212-216. https://doi.org/10.1038/s41586-018-0085$\underline{3}$

Tressoldi, P. E., Pederzoli, L., Caini, P., Ferrini, A., Melloni, S., Prati, E., ... \& Trabucco, A. (2015). Hypnotically induced out-of-body experience: How many bodies are there? Unexpected discoveries about the subtle body and psychic body. SAGE Open, 5(4), 2158244015615919 .

Tressoldi, P., \& Storm, L. (2020). Nonlocal Perception: A Synthesis of the Meta-Analytic Evidence. PsyArxiv; https://doi.org/10.31234/osf.io/5gwqb

Tressoldi, P. E., \& Storm, L. (2021). Stage 2 Registered Report: Anomalous perception in a Ganzfeld condition-A meta-analysis of more than 40 years investigation. F1000Research, 10(234), 234. https://doi.org/10.12688/f1000research.51746.1)

Tressoldi, P., \& Facco, E. (2021). Why Consciousness is primary: epistemological and scientific evidence. MindRxiv; https://doi.org/10.31231/osf.io/2nwb6

Yaden, D. B., Eichstaedt, J. C., Schwartz, H. A., Kern, M. L., Le Nguyen, K. D., Wintering, N. A., Hood, R. W., Jr., \& Newberg, A. B. (2016). The language of ineffability: Linguistic analysis of mystical experiences. Psychology of Religion and Spirituality, 8(3), 244-252. https://doi.org/10.1037/rel0000043

Wahbeh, H., Sagher, A., Back, W., Pundhir, P., \& Travis, F. (2018). A systematic review of transcendent states across meditation and contemplative traditions. Explore, 14(1), 19-35. https://doi.org/10.1016/j.explore.2017.07.007

Wikipedia contributors. (2021). Advaita Vedanta. In Wikipedia, The Free Encyclopedia. Retrieved, April 25, 2021, from https://en.wikipedia.org/w/index.php?title=Advaita_Vedanta\&oldid=1016651250

Wiseman, H. (2015). Death by experiment for local realism. Nature, 526(7575), 649-650. 
Wolchover, N. (2020). What is a particle. Quanta Magazine, November 12;

https://www.quantamagazine.org/what-is-a-particle-20201112 